\title{
CXCL13 Levels Indicate Treatment Responsiveness to Fingolimod in MS Patients
}

\author{
Zerrin Karaaslan $^{a}$ Murat Kurtuncu $^{\text {b }}$ Halil İbrahim Akcay ${ }^{b}$ Tuncay Gunduz $^{b}$ \\ Burcu Altunrende ${ }^{c}$ Recai Turkoglu ${ }^{d}$ Mefkure Eraksoy ${ }^{b}$ Canan Ulusoy $^{a}$ \\ Vuslat Yilmaz $^{a}$ Erdem Tüzün $^{a}$ \\ aDepartment of Neuroscience, Aziz Sancar Institute of Experimental Medicine, Istanbul University, Istanbul, Turkey; \\ ${ }^{\mathrm{b}}$ Department of Neurology, Istanbul Faculty of Medicine, Istanbul University, Istanbul, Turkey; 'Department of

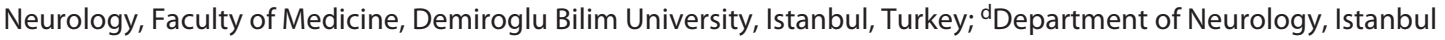 \\ Haydarpasa Numune Training and Research Hospital, Istanbul, Turkey
}

Dear Editor,

Multiple sclerosis (MS) is a chronic demyelinating autoimmune disease of the central nervous system (CNS) induced by the complex interplay between the innate and adaptive immune systems [1]. Fingolimod, an oral drug that has been approved as a disease-modifying therapy in MS, acts through the sphingosine-1-phosphate receptor and inhibits the egress of $\mathrm{B}$ and $\mathrm{T}$ cells from lymph nodes. In addition to reducing the circulating lymphocytes in the peripheral blood, it also regulates subsets of immune cells [2]. In our previous study conducted with 66 relapsing remitting MS patients ( $35.7 \pm 8.2$ years old; 46 women), we investigated the effect of fingolimod treatment on serum cytokine and chemokine levels. Included relapsing remitting MS patients were either unable to tolerate or nonresponders to first-line treatments (interferon- $\beta$, glatiramer acetate, or teriflunomide). None of the patients had a relapse or had received immunosuppressive or immunomodulating agents in the last 3 months. Detailed inclusion and exclusion criteria are provided elsewhere [3]. The study protocol was approved by the institutional review board, and all subjects provided written informed consent. Sera were obtained between 08:00 and 10:00 a.m. 1 day before and 3 and 6 months after fingoli-

karger@karger.com

(c) 2021 S. Karger AG, Basel

www.karger.com/ene

Karger! mod treatment $(0.5 \mathrm{mg} /$ day $)$ and kept at $-80^{\circ} \mathrm{C}$ until assayed. Serum cytokine and chemokine levels were measured by multiplex immunoassays (Invitrogen, Carlsbad, CA, USA). In this previous study, fingolimod was found to cause a significant elevation of serum CCL2 and CCL5 levels [3].

Our next aim was to investigate whether baseline (prefingolimod treatment) levels of cytokines could be used to predict nonresponsiveness to fingolimod treatment, which was defined as having $\geq 1.0$ point increase in the Expanded Disability Status Scale (EDSS) score and/or experiencing $>1$ attack under 2 years of fingolimod treatment. For this purpose, 17 of 66 patients with a 2 -year follow-up duration were included in the present study, and 5 of these patients were determined to fulfill the nonresponder criteria. Clinical and demographic features and baseline cytokine levels of responder and nonresponder patients were compared using the Mann-Whitney $U$ and $\chi^{2}$ tests, as required (Table 1 ). Correlation analysis was done with Spearman's test. A $p$ value of $<0.05$ was considered statistically significant. Among IL-4, IL-17A, IL-22, IL-23, IFN- $\gamma$, TNF- $\alpha$, IL-8, CCL2 (MCP-1), CCL5 (RANTES), CXCL10 (IP-10), and CXCL13, only baseline CXCL13 levels were found to be significantly higher in 
Table 1. Clinical and demographic characteristics and serum cytokine profiles of fingolimod-responder and nonresponder MS patients

\begin{tabular}{lccc}
\hline & $\begin{array}{l}\text { Fingolimod } \\
\text { responders } \\
(n=12)\end{array}$ & $\begin{array}{l}\text { Fingolimod } \\
\text { nonresponders } \\
(n=5)\end{array}$ & $p$ value \\
\hline Sex (female/male) & $8 / 4$ & $5 / 0$ & 0.261 \\
Age & $33.75 \pm 9.27$ & $32.80 \pm 8.56$ & 0.847 \\
Disease onset & $26.50 \pm 7.66$ & $26.40 \pm 6.35$ & 0.980 \\
Duration of MS & $7.25 \pm 5.03$ & $6.40 \pm 5.23$ & 0.560 \\
Relapse number in the last 2 years & $0.75 \pm 0.62$ & $1.40 \pm 1.16$ & 0.506 \\
Follow-up time, years & $3.40 \pm 1.80$ & $3.79 \pm 0.28$ & 0.252 \\
Attacks under fingolimod, $n$ & $0.17 \pm 0.11$ & $1.80 \pm 0.37$ & 0.001 \\
EDSS at the first visit & $1.62 \pm 1.11$ & $1.5 \pm 0.71$ & 0.745 \\
EDSS at the last visit & $1.66 \pm 1.15$ & $3.6 \pm 2.04$ & 0.004 \\
CXCL13, pg/mL & $58.25 \pm 27.45$ & $127.2 \pm 46.13$ & 0.009 \\
CXCL10, ng/mL & $9.29 \pm 1.25$ & $17.47 \pm 5.23$ & 0.196 \\
CCL5, ng/mL & $318.05 \pm 36.93$ & $329.76 \pm 65.21$ & 0.871 \\
CCL2, ng/mL & $38.25 \pm 11.18$ & $58.33 \pm 11.53$ & 0.064 \\
IL-4, pg/mL & $2.09 \pm 0.19$ & $2.35 \pm 0.11$ & 0.234 \\
IL-8, pg/mL & $1.35 \pm 0.51$ & $2.01 \pm 0.82$ & 0.506 \\
IL-17A, pg/mL & $1.09 \pm 0.05$ & $1.17 \pm 0.05$ & 0.279 \\
IL-22, pg/mL & $22.76 \pm 1.18$ & $20.96 \pm 1.79$ & 0.419 \\
TNF- $\alpha, \mathrm{pg} / \mathrm{mL}$ & $0.84 \pm 0.19$ & $1.43 \pm 0.31$ & 0.064 \\
\hline
\end{tabular}

Numerical data are denoted as mean \pm standard deviation. EDSS, Expanded Disability Status Scale; MS, multiple sclerosis.

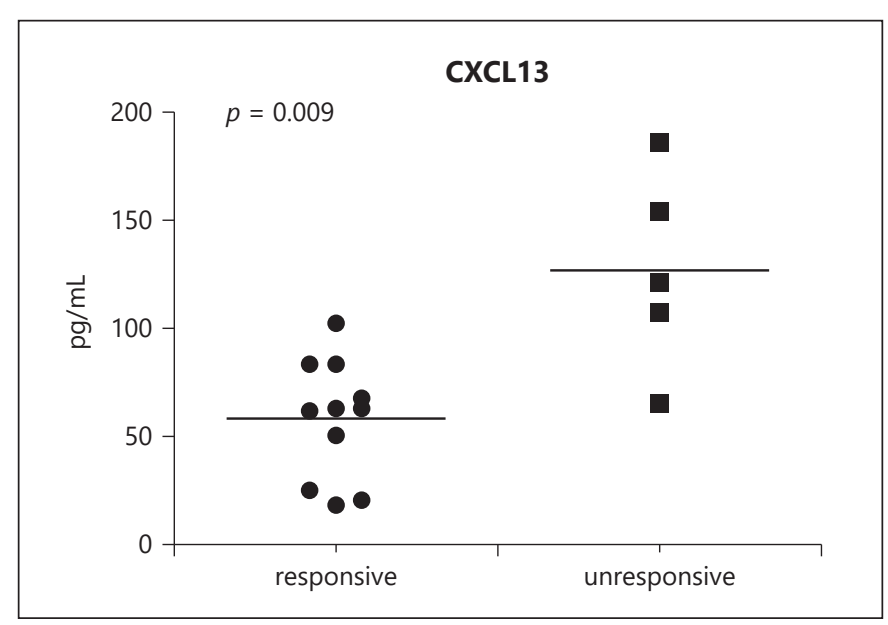

Fig. 1. Serum CXCL13 levels of fingolimod-responsive and -unresponsive MS patients. MS, multiple sclerosis.

nonresponder patients compared to responder ones $(127.2 \pm 46.13$ vs. $58.25 \pm 27.45 \mathrm{pg} / \mathrm{mL}, p=0.009)(\mathrm{Ta}-$ ble 1) (shown in Fig. 1).

These results suggest that having an elevated pretreatment serum level of CXCL13 might lead to a poor re- sponse to fingolimod treatment. Notably, responder and nonresponder MS patients had identical clinical demographic features, relapse rates, and disability levels prior to fingolimod treatment. Among 5 nonresponder patients, 3 of them also did not show effective response to first-line treatments (interferon- $\beta$ and glatiramer acetate) before fingolimod. The substantial increase of attack numbers and EDSS scores under fingolimod treatment in the nonresponder group (Table 1) suggests that fingolimod treatment might even be exhibiting an adverse and worsening action in MS patients with elevated CXCL13 levels.

CXCL13 is a chemo-attractant molecule that is primarily expressed in lymphoid organs and controls the recruitment and compartmentalization of lymphocytes, particularly B cells [4]. Presence of meningeal B-cell follicles in MS suggests a germinal center reaction in the CNS of MS patients [5]. Increased levels of CXCL13, one of the mediators of germinal center formation, was found to be correlated with disease activity in MS patients, which might indicate the importance of recruitment of B cells to the CNS in the pathogenesis of MS [6]. In a model investigating lymphocyte migration, sphingosine1-phosphate stimulation was found to be necessary to 
overcome the lymphocyte-recruiting effect of CXCL13, which is required for the positioning of marginal zone $\mathrm{B}$ cells in splenic follicles [7]. Thus, high levels of CXCL13 in MS patients might overcome the inhibitory effect of fingolimod on the egress of B cells from lymphoid tissues, thereby causing a poor response to fingolimod treatment. Moreover, the findings that revealed decrease in the levels of CXCL13 by treatment with rituximab as well as other disease-modifying therapies suggested that CXCL13 may have key roles in MS inflammation other than B-cell regulation [8].

Peripheral blood T-cell subsets determined by flow cytometry have also been reported to predict responsiveness to fingolimod treatment [9]. We herein propose for the first time that serum CXCL13 levels might also be used for the same purpose without undergoing the hardship of isolating mononuclear cells from peripheral blood and dealing with standardization procedures of flow cytometry. This notion is supported by previous reports suggesting that pretreatment CXCL13 levels have a predictive value for therapeutic responsiveness in MS patients treated with rituximab and natalizumab $[6,10]$.

Notably, nonresponder patients showed trends toward exhibiting increased serum CCL2 and TNF- $\alpha$ levels, as well. CCL2 and TNF- $\alpha$ are inflammatory cytokines highly required for accumulation of inflammatory cells in the MS brain [11]. Interestingly, fingolimod treatment increases serum levels of CCL 2 and peripheral blood proportions of TNF- $\alpha$-producing T cells [3]. These poten- tially hazardous side effects of fingolimod treatment might adversely affect the disease course in a subgroup of MS patients. Future analysis with a larger cohort might shed further light on the significance of these cytokines as a biomarker for fingolimod responsiveness.

MS is a chronic disease which requires life-long treatment. Choosing an optimal treatment is important for both patients and the health system. Thus, it is important to predict the response to treatment options. Although it has been carried out on a small cohort, with this observational study, we suggest that CXCL13 could be a promising biomarker that predicts response to fingolimod treatment.

\section{Conflict of Interest Statement}

The authors declare no conflicts of interest.

\section{Funding Sources}

This study was sponsored by Novartis Pharmaceuticals.

\section{Author Contributions}

M.K., H.I.A., B.A., R.T., and M.E. collected the data; Z.K., C.U., V.Y., and E.T. performed the experimental procedure; Z.K., V.Y., and E.T. drafted the work; M.K., M.E., and E.T. gave final approval.

\section{References}

1 Yadav SK, Mindur JE, Ito K, Dhib-Jalbut S. Advances in the immunopathogenesis of multiple sclerosis. Curr Opin Neurol. 2015; 28(3):206-19.

2 Claes N, Dhaeze T, Fraussen J, Broux B, Van Wijmeersch B, Stinissen P, et al. Compositional changes of $\mathrm{B}$ and $\mathrm{T}$ cell subtypes during fingolimod treatment in multiple sclerosis patients: a 12-month follow-up study. PLoS One. 2014;9(10):e111115.

3 Kurtuncu M, Yilmaz V, Akcay HI, Turkoglu $\mathrm{R}$, Altunrende B, Cinar SA, et al. Impact of fingolimod on $\mathrm{CD} 4+\mathrm{T}$ cell subset and cytokine profile of relapsing remitting multiple sclerosis patients. J Neuroimmunol. 2019; 337:577065.

4 Huber AK, Irani DN. Targeting CXCL13 during neuroinflammation. Adv Neuroimmune Biol. 2015;6(1):1-8.
5 Magliozzi R, Howell O, Vora A, Serafini B, Nicholas R, Puopolo M, et al. Meningeal Bcell follicles in secondary progressive multiple sclerosis associate with early onset of disease and severe cortical pathology. Brain. 2007; 130(Pt 4):1089-104.

6 Sellebjerg F, Börnsen L, Khademi M, Krakauer M, Olsson T, Frederiksen JL, et al. Increased cerebrospinal fluid concentrations of the chemokine CXCL13 in active MS. Neurology. 2009;73(23):2003-10.

7 Cinamon G, Matloubian M, Lesneski MJ, Xu Y, Low C, Lu T, et al. Sphingosine 1-phosphate receptor 1 promotes $\mathrm{B}$ cell localization in the splenic marginal zone. Nat Immunol. 2004;5(7):713-20.

8 Novakova L, Axelsson M, Malmeström C, Zetterberg H, Blennow K, Svenningsson A, et al. NFL and CXCL13 may reveal disease activity in clinically and radiologically stable MS. Mult Scler Relat Disord. 2020;46:102463.
9 Ghadiri M, Rezk A, Li R, Evans A, Giacomini PS, Barnett MH, et al. Pre-treatment T-cell subsets associate with fingolimod treatment responsiveness in multiple sclerosis. Sci Rep. 2020;10(1):356.

10 Alvarez E, Piccio L, Mikesell RJ, Trinkaus K, Parks BJ, Naismith RT, et al. Predicting optimal response to $\mathrm{B}$-cell depletion with rituximab in multiple sclerosis using CXCL13 index, magnetic resonance imaging and clinical measures. Mult Scler J Exp Transl Clin. 2015; 1:205521731562380.

11 Lomakin Y, Belogurov A Jr, Glagoleva I, Stepanov A, Zakharov K, Okunola J, et al. Administration of myelin basic protein peptides encapsulated in mannosylated liposomes normalizes level of serum TNF- $\alpha$ and IL-2 and chemoattractants CCL2 and CCL4 in multiple sclerosis patients. Mediators Inflamm. 2016;2016:2847232. 